\title{
Bounds for the Perron Root of Irreducible Nonnegative Matrices
}

\author{
Liao Ping ${ }^{1}$ \\ ${ }^{1}$ Sichuan Vocational and Technical College, Suining, Sichuan, China \\ Correspondence: Liao Ping, Sichuan Vocational and Technical College, Suining, Sichuan, China. E-mail: hen- \\ rycq@163.com
}

Received: January 29, 2015 Accepted: February 10, 2015 Online Published: February 24, 2015

doi:10.5539/jmr.v7n1p97 URL: http://dx.doi.org/10.5539/jmr.v7n1p97

\section{Abstract}

Some bounds for the Perron root $\rho$ of positive matrices are proposed. We proved that

$$
\max _{1 \leq i \leq n}\left(\sum_{k} a_{i k} m_{k i}\right) \leq \rho \leq \min _{1 \leq i \leq n}\left(\sum_{k} a_{i k} M_{k i}\right) .
$$

where

$$
m_{k i}=\min _{t} \frac{\left(\alpha_{k}, \beta_{t}\right)}{\left(\alpha_{i}, \beta_{t}\right)}, M_{k i}=\max _{t} \frac{\left(\alpha_{k}, \beta_{t}\right)}{\left(\alpha_{i}, \beta_{t}\right)}
$$

and $\alpha_{k}$ denotes the $k$-th row of matrix $A, \beta_{t}$ the $t$-th column of $A,\left(\alpha_{k}, \beta_{t}\right)$ denotes the inner product of $\alpha_{k}$ and $\beta_{t}$. And these bounds can also be used to estimate the Perron root of nonnegative irreducible matrices.

Keywords: Perron root, nonnegative matrix, lower bound, irreducible

\section{Introduction}

Let $A=\left(a_{i j}\right)$ be an irreducible nonnegative matrix of order $n$, the Perron root $\rho$ of $A$ is a positive real eigenvalue of $A$ and any other eigenvalue $\lambda$ is strictly smaller than $\rho$ in absolute value $(|\lambda|<\rho)$. It is also called the PerronFrobenius eigenvalue, the greatest characteristic root of $A$ or the greatest latent root of $A$. For the bounds of Perron root ,the following results are well known

$$
\min _{i} r_{i} \leq \rho \leq \max _{i} r_{i}
$$

here $r_{i}$ is the $i$-th row sum. For nonnegative matrices with nonzero row sums $r_{1}, r_{2}, \ldots, r_{n}$, the bounds in (1) were improved by Minc (Minc, 1988) as follows:

$$
\min _{i} \frac{1}{r_{i}} \sum_{j=1}^{n} a_{i j} r_{j} \leq \rho \leq \max _{i} \frac{1}{r_{i}} \sum_{j=1}^{n} a_{i j} r_{j} .
$$

This result was further generalized by Liu (Shulin Liu,1996). For positive matrices( $\left.a_{i j}>0, i, j=1,2, \ldots, n\right)$, formula (1) were also improved by A.Ostrowski (A. Ostrowski, 1952) and by A.Brauer (Brauer A., 1957). In the paper of Brauer A. (Brauer A., 1974), the following bounds were obtained.

$$
\min _{i \neq j} M(i, j) \leq \rho \leq \max _{i \neq j} M(i, j) .
$$

where $M(i, j)=\frac{1}{2}\left(a_{i i}+a_{j j}+\left[\left(a_{i i}-a_{j j}\right)^{2}+4 P_{i} P_{j}\right]^{\frac{1}{2}}\right)$, and $P_{i}=\sum_{j \neq i} a_{i j}$. There are other bounds which are obtained by some special way (Linzhang Lu, 2002).

In this paper, some new bounds for the Perron root of positive matrices are proposed at first, and these bounds can also be used to estimate the Perron root of irreducible nonnegative matrix.

\section{The Main Results}

In this section, we will get some new bounds for positive matrices at first. In order to get our results, we propose some lemmas. 
Lemma 1 (Shulin Liu, 1996)Let $p_{k}>0, q_{k}>0, k=1,2, \ldots, n$, then

$$
\min _{k} \frac{p_{k}}{q_{k}} \leq \frac{\sum_{k} p_{k}}{\sum_{k} q_{k}} \leq \max _{k} \frac{p_{k}}{q_{k}}
$$

Lemma 2 Let $A=\left(a_{i j}\right)_{n \times n}$ be a positive matrix of order $n$ and $\rho$ its Perron root, Suppose $x=\left(x_{1}, x_{2}, \ldots, x_{n}\right)$ is the positive eigenvector of $\rho, x_{i}$ and $x_{j}$ are two arbitrarily elements of $x$, then

$$
\min _{k} \frac{a_{j k}}{a_{i k}} \leq \frac{x_{j}}{x_{i}} \leq \max _{k} \frac{a_{j k}}{a_{i k}}
$$

Proof. If $x$ is the positive eigenvector of $\rho$, then consider the $j$-th and $i$-th elements of the equation $A x=\rho x$, one can get

$$
\frac{x_{j}}{x_{i}}=\frac{\rho x_{j}}{\rho x_{i}}=\frac{\sum_{k} a_{j k} x_{k}}{\sum_{k} a_{i k} x_{k}}
$$

and by lemma 1 we know (6) holds and the proof is complete.

Now we give some bounds for the Perron root of positive matrices.

Theorem 1 Let $A=\left(a_{i j}\right)_{n \times n}$ be a positive matrix of order $n$ and $\rho$ its Perron root, then

$$
\max _{1 \leq i \leq n}\left(\sum_{k} a_{i k} m_{k i}\right) \leq \rho \leq \min _{1 \leq i \leq n}\left(\sum_{k} a_{i k} M_{k i}\right)
$$

where

$$
m_{k i}=\min _{t} \frac{a_{k t}}{a_{i t}}, M_{k i}=\max _{t} \frac{a_{k t}}{a_{i t}} .
$$

Proof. Suppose $x$ is the positive eigenvector of $\rho$, then consider an arbitrarily element of equation $A x=\rho x$, suppose the $i$-th element, we can get

$$
\rho x_{i}=\sum_{k=1}^{n} a_{i k} x_{k}
$$

that is

$$
\rho=\sum_{k=1}^{n} a_{i k} \frac{x_{k}}{x_{i}}
$$

From the $k$-th and the $i$-th elements of the equation $A x=\rho x$, by lemma 2, we have

$$
\min _{t} \frac{a_{k t}}{a_{i t}} \leq \frac{x_{k}}{x_{i}} \leq \max _{t} \frac{a_{k t}}{a_{i t}} .
$$

Let

$$
m_{k i}=\min _{t} \frac{a_{k t}}{a_{i t}}, M_{k i}=\max _{t} \frac{a_{k t}}{a_{i t}}
$$

then

$$
\sum_{k} a_{i k} m_{k i} \leq \rho \leq \sum_{k} a_{i k} M_{k i}
$$

Because the arbitrariness of $i$, so we get

$$
\max _{1 \leq i \leq n}\left(\sum_{k} a_{i k} m_{k i}\right) \leq \rho \leq \min _{1 \leq i \leq n}\left(\sum_{k} a_{i k} M_{k i}\right) .
$$

The proof is complete.

This upper and lower bounds sometimes are not good enough because of the rough estimate of $\frac{x_{k}}{x_{i}}$. For example, consider the following matrix

$$
A=\left(\begin{array}{lll}
1 & 1 & 2 \\
2 & 1 & 3 \\
2 & 3 & 5
\end{array}\right) .
$$


We just get $\frac{20}{3} \leq \rho \leq 9$ by (9), though this bound is better than (1), It is worse than many other bounds obtained by (2),(3) and so on, so we have to improve this result to get closer bounds. Note the fact that $A$ and $A^{m}$ have the same eigen-space, so $A^{m}$ can also be used to estimate $\frac{x_{k}}{x_{i}}$. The following lemma will show their relations.

Lemma 3 Let $A=\left(a_{i j}\right)_{n \times n}$ be a positive matrix of order $n$ and $\rho$ its Perron root, Suppose $x=\left(x_{1}, x_{2}, \ldots, x_{n}\right)$ is the positive eigenvector of $\rho, x_{i}$ and $x_{j}$ are two arbitrarily elements of $x$, and $A^{m}=\left(a_{i k}^{(m)}\right)_{n \times n}$, then

$$
\min _{k} \frac{a_{j k}^{(m)}}{a_{i k}^{(m)}} \leq \min _{k} \frac{a_{j k}^{(m+1)}}{a_{i k}^{(m+1)}} \leq \frac{x_{j}}{x_{i}} \leq \max _{k} \frac{a_{j k}^{(m+1)}}{a_{i k}^{(m+1)}} \leq \max _{k} \frac{a_{j k}^{(m)}}{a_{i k}^{(m)}} .
$$

Proof. If $x$ is the positive eigenvector of $\rho$, then consider the $j$-th and $i$-th elements of the equation $A^{m+1} x=\rho^{m+1} x$. Because $a_{j k}^{(m+1)}=\sum_{t} a_{j t}^{(m)} a_{t k}, a_{i k}^{(m+1)}=\sum_{t} a_{i t}^{(m)} a_{t k}$, so we have

$$
\frac{x_{j}}{x_{i}}=\frac{\rho^{m+1} x_{j}}{\rho^{m+1} x_{i}}=\frac{\sum_{k} a_{j k} a_{j k}^{(m+1)} x_{k}}{\sum_{k} a_{i k}^{(m+1)} x_{k}}
$$

by lemma 1 we get

$$
\min _{k} \frac{a_{j k}^{(m)}}{a_{i k}^{(m)}} \leq \min _{k} \frac{a_{j k}^{(m+1)}}{a_{i k}^{(m+1)}} \leq \frac{x_{j}}{x_{i}} \leq \max _{k} \frac{a_{j k}^{(m+1)}}{a_{i k}^{(m+1)}} \leq \max _{k} \frac{a_{j k}^{(m)}}{a_{i k}^{(m)}}
$$

The proof is complete.

Lemma 3 shows that use $A^{m+1}$ to estimate $\frac{x_{k}}{x_{i}}$ will always work better than $A^{m}$. If one wants to get a sharper bound, then we just need to choose a bigger $m$. We proposed the improved estimation as follows.

Theorem 2 Let $A=\left(a_{i j}\right)_{n \times n}$ be a positive matrix of order $n$ and $\rho$ its Perron root, and $A^{m}=\left(a_{i k}^{(m)}\right)_{n \times n}$, then

$$
\max _{1 \leq i \leq n}\left(\sum_{k} a_{i k} m_{k i}^{\prime}\right) \leq \rho \leq \min _{1 \leq i \leq n}\left(\sum_{k} a_{i k} M_{k i}^{\prime}\right)
$$

where

$$
m_{k i}^{\prime}=\min _{t} \frac{a_{k t}^{(m)}}{a_{i t}^{(m)}}, M_{k i}^{\prime}=\max _{t} \frac{a_{k t}^{(m)}}{a_{i t}^{(m)}} .
$$

One step further, we can also have the following results.

Theorem 3 Let $A=\left(a_{i j}\right)_{n \times n}$ be a positive matrix of order $n$ and $\rho$ its Perron root, and $A^{m}=\left(a_{i k}^{(m)}\right)_{n \times n}$, then

$$
\max _{1 \leq i \leq n}\left(\sum_{k} a_{i k}^{(m)} m_{k i}^{\prime}\right)^{\frac{1}{m}} \leq \rho \leq \min _{1 \leq i \leq n}\left(\sum_{k} a_{i k}^{(m)} M_{k i}^{\prime}\right)^{\frac{1}{m}} .
$$

where $m_{k i}^{\prime}, M_{k i}^{\prime}$ is the same as theorem 2.

Proof. Just consider the $i$-th element of the equation $A^{m} x=\rho^{m} x$,

$$
\rho^{m} x_{i}=\sum_{k=1}^{n} a_{i k}^{(m)} x_{k}
$$

then

$$
\rho=\left(\sum_{k=1}^{n} a_{i k}^{(m)} \frac{x_{k}}{x_{i}}\right)^{\frac{1}{m}}
$$

together with lemma 3 we get (13).

Take $m=2$, then we get the following corollary.

Corollary 1 Let $A=\left(a_{i j}\right)_{n \times n}$ be a positive matrix of order $n$ and $\rho$ its Perron root, then

$$
\max _{1 \leq i \leq n}\left(\sum_{k} a_{i k} m_{k i}^{\prime \prime}\right) \leq \rho \leq \min _{1 \leq i \leq n}\left(\sum_{k} a_{i k} M_{k i}^{\prime \prime}\right) .
$$


where

$$
m_{k i}^{\prime \prime}=\min _{t} \frac{\left(\alpha_{k}, \beta_{t}\right)}{\left(\alpha_{i}, \beta_{t}\right)}, M_{k i}^{\prime \prime}=\max _{t} \frac{\left(\alpha_{k}, \beta_{t}\right)}{\left(\alpha_{i}, \beta_{t}\right)}
$$

and $\alpha_{k}$ denotes the $k$-th row of $A, \beta_{t}$ the $t$-th column of $A,\left(\alpha_{k}, \beta_{t}\right)$ denotes the inner product of $\alpha_{k}$ and $\beta_{t}$.

Corollary 2 Let $A=\left(a_{i j}\right)_{n \times n}$ be a positive matrix of order $n$ and $\rho$ its Perron root, then

$$
\max _{1 \leq i \leq n}\left(\sum_{k} a_{i k} m_{k i 1}\right) \leq \rho \leq \min _{1 \leq i \leq n}\left(\sum_{k} a_{i k} M_{k i 1}\right) .
$$

where

$$
m_{k i 1}=\lim _{m \rightarrow+\infty} m_{k i}^{\prime}, M_{k i 1}=\lim _{m \rightarrow+\infty} M_{k i}^{\prime}
$$

and $m_{k i}^{\prime}, M_{k i}^{\prime}$ is the same as theorem 2 .

Proof. By lemma 3, we know that $m_{k i}^{\prime}, M_{k i}^{\prime}$ is both bounded and monotonic, So the limit exists, and by theorem 2 we get (15).

Corollary 3 Let $A=\left(a_{i j}\right)_{n \times n}$ be a positive matrix of order $n$ and $\rho$ its Perron root, then

$$
\max _{1 \leq i \leq n}\left(\sum_{k} a_{i k} m_{k i 1}\right)^{\frac{1}{m}} \leq \rho \leq \min _{1 \leq i \leq n}\left(\sum_{k} a_{i k} M_{k i 1}\right)^{\frac{1}{m}} .
$$

where $m_{k i 1}, M_{k i 1}$ is the same as corollary 2.

Now we focus on an irreducible nonnegative matrix $A$. If there are no zero entries in the matrix $A$, then it is a positive matrix, the bounds proposed above can be used directly. If there are zero entries in the matrix, the above results can not be used directly, one have to make a little change. Since for an irreducible nonnegative matrix $A$, let $B=(A+I)^{n-1}$, then $B>0$. So one can use the above theorems to estimate the Perron root of matrix $B$, and then by the relation of matrix $A$ and $B$ 's Perron root $\rho(B)=(\rho(A)+1)^{n-1}$, one can get the bounds for the Perron root of matrix $A$.

Remark 1 The above methods can also be used on the transpose matrix of $A$.

\section{Some Examples}

Example 1 Suppose

$$
A=\left(\begin{array}{lll}
1 & 1 & 2 \\
2 & 1 & 3 \\
2 & 3 & 5
\end{array}\right)
$$

By (1) we get $4 \leq \rho \leq 10$, by (2) $7.3333 \leq \rho \leq 7.6$, by (3) $4.8730 \leq \rho \leq 8.3852$, by (7) $6.6667 \leq \rho \leq 9.000$, by (14) we can get $7.4444 \leq \rho \leq 7.6000$. Let $m=5$, by (12) we can get 7.53112 $\leq \rho \leq 7.53116$, by (13) we can even get $7.53112 \leq \rho \leq 7.53113$. And its not difficult to compute that the Perron root of $A$ is $7.53112 \cdots$.

\section{Example 2 Suppose}

$$
A=\left(\begin{array}{llllll}
1 & 1 & 1 & 1 & 1 & 1 \\
1 & 2 & 2 & 2 & 2 & 2 \\
1 & 2 & 3 & 3 & 3 & 3 \\
1 & 2 & 3 & 4 & 4 & 4 \\
1 & 2 & 3 & 4 & 5 & 5 \\
1 & 2 & 3 & 4 & 5 & 6
\end{array}\right) .
$$

By (1) we get $6 \leq \rho \leq 21$, by (2) $15.1667 \leq \rho \leq 17.6667$, by (3) $8.2268 \leq \rho \leq 19.5258$, by (14) we can get $17 \leq \rho \leq 17.6667$. Let $m=5$, by (12) we can get $17.2066 \leq \rho \leq 17.2076$, by (13) we can even get $17.2068 \leq \rho \leq 17.2070$. And the Perron root of $A$ is $17.20686 \cdots$.

Example 3 Suppose

$$
A=\left(\begin{array}{lll}
1 & 1 & 2 \\
2 & 1 & 3 \\
2 & 0 & 5
\end{array}\right)
$$


Consider the matrix $B=(A+I)^{2}$, then $B$ is a positive matrix. Let $m=3$, by (12) we get $50.3896 \leq \rho(B) \leq 50.5998$, since $\rho(A)=\sqrt{\rho(B)}-1$, then we can get $6.0986 \leq \rho(A) \leq 6.1134$. And the Perron root of $A$ is $6.0996 \cdots$.

\section{References}

Alfred, B., \& Ivey, C. G. (1974). Bounds for the greatest characteristic root of an irreducible nonnegative matrix. Linear Algebra and its Application, 105-107.

Brauer, A. (1952). Bounds for the greatest latent root of a positive matrix. Journal of London Mathematics Society, 253-256.

Brauer, A. (1957). The theorems of Ledermann and Ostrowski on positive matrices. Duke Math J., 265-274.

Liu, S. (1996). Bounds for the greatest characteristic root of a nonnegative matrix. Linear Algebra and its Application, 151-160.

Lu, L. (2002).Perron complement and perron root. Linear Algebra and its Application, 239-248.

Minc, H. (1988). Nonnegative Matrices. Wiley, New York.

\section{Copyrights}

Copyright for this article is retained by the author(s), with first publication rights granted to the journal.

This is an open-access article distributed under the terms and conditions of the Creative Commons Attribution license (http://creativecommons.org/licenses/by/3.0/). 